\title{
Forecasting Gas Production Rate in Underground Gas Reservoirs Using Artificial Neural Networks
}

\author{
Morteza Bagherpour ${ }^{1, *}$, MH.Bagherpour ${ }^{2}$, K. Roodani ${ }^{2}$ \\ ${ }^{1}$ Department of industrial Engineering, Iran University of Science and Technology, Iran \\ ${ }^{2}$ JondiShapour Company, Shiraz, Iran \\ *Corresponding Author: mortezabagherpour@gmail.com
}

Copyright (C) 2014 Horizon Research Publishing All rights reserved.

\begin{abstract}
Underground Gas Reservoir (UGS) is one of the important types of reservoirs which after depletion, a significant amount of gas will inject into the reservoir. Then, in winter and in emergency cases, the stored gas will transfer to consumption centers. Here, forecasting of gas production rate will play an important role to discover when the reservoir will be depleted and, how much stored gas amount will be then injected. The forecasting procedure of UGS highly depends on affecting factors such as pressure, gas in place, bulk volume and etc. Due to complexity and non-linearity manner of input and output variables, an intelligent data driven modeling procedure is useful to apply. In this paper, therefore, a Multi Layer Perceptron (MLP) neural network is applied to predict the production rate. Also, a time series model is conducted to predict time based variables. The results show a 99 percent of accuracy which demonstrate superiority of the proposed approach over existing traditional models.
\end{abstract}

Keywords UGS, Gas Production Rate, Forecasting, Neural Networks, MLP, Time Series Analysis

\section{Introduction and Literature Survey}

Common type of storage is depleted natural gas or oil fields which are close to consumption centers. Conversion of a reservoir from production to storage has some advantages of existing wells, gathering systems, and pipeline connections. Generally, depleted oil and gas reservoirs are the most commonly used underground storage sites [1].

As long as a reservoir depleted, it is somehow possible to store gas in the reservoir. This process is performed by pipeline. However, the pipelines have some limitations through transferring of significant gas amount. Therefore, the behavior of gas production rate has a significant impact on further calculations of working gas to be stored. The amount of stored gas, when required can be depleted and transferred by pipelines to consumption centers.

Forecasting of gas production rate however depends on some affecting parameters such as [2]:

- Total gas storage capacity

- Total gas in storage

- Working gas capacity

- Cycling rate

- Deliverability

- Injection capacity

Most of the researches in the area of gas reservoirs focused on natural gas reservoirs which normally applied reservoir simulation modeling in order to predict behavior of the well and further possible scenarios developed $[3,4]$. Recently, modern intelligent well technologies such as artificial neural networks have been partially concentrated by some researchers. Most of the intelligent well technologies applied in natural gas reservoirs. For instance, Ayala et al. [5] applied a neuro simulation model for exploration. Then Mann and Ayala [6] applied a neural network for design and optimization of natural gas storage.

After going through the literature, it has been proved although artificial neural networks have been extensively applied in many sciences and technologies; however, it has been rarely incorporated through gas condensate reservoirs. To the best of our knowledge, application of artificial neural networks in underground gas condensate reservoirs for forecasting of gas production rate (depletion rate) has not been found in the literature which is concentrated in this paper originally. Also, a comparative analysis between potential neural networks has been examined for selecting the best known neural network which has not been found in the literature.

\section{Problem Statement}

Here seven well production trends have been considered. The initial gas in place, bulk volume, period of time, and production rate are incorporated as inputs. Bottom hole pressure is considered as output. A series of data including more than 120 months have been incorporated through modeling procedure. The production rate has a fluctuation in trend due to season and pressure drop. As time passes, the 
pressure trend declines. The initial pressure was 350 bar which finally reservoir abandonment will take place at 130 bar. Here, it is under investigation that when well abandonment will take place, what the pressure is at each specific period and finally how much is the production rate at each period. Therefore, inputs and output are highly dependent and there is a relationship among the variables. This case, maybe happen in several production gas reservoirs similarly. If so, the tuning procedure based on available data is required to perform.

\section{Modeling Procedure}

\subsection{Neural Network Modeling}

The architecture of the neural network has been shown in figure 1

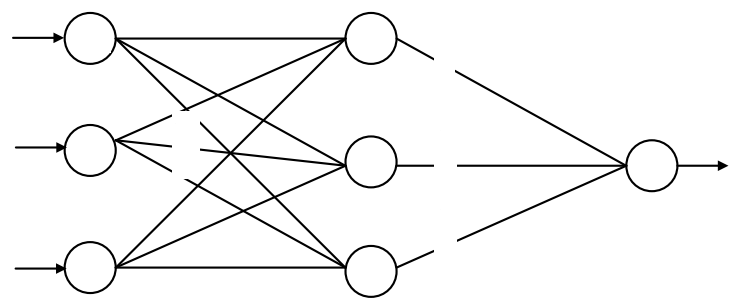

Figure1. Architecture of neural network

The input and output relations of neural network's units of above figure are as the followings:

- Inputs units:

$$
O_{p i}=X_{p i}, i=1,2, \ldots, n
$$

- Hidden units:

$$
\begin{array}{r}
O_{p j}=f\left(N e t_{p j}\right),(j=1,2, \ldots, m) \\
N e t_{p j}=\sum_{i=1}^{n} W_{i j} \cdot O_{p i}+B_{j}
\end{array}
$$

Output units:

$$
\begin{aligned}
& O_{p}=f\left(\mathrm{Net}_{p}\right) \\
& N e t_{p}=\sum_{j=1}^{m} V_{j} \cdot O_{p j}+B
\end{aligned}
$$

$\mathrm{P}$ is the number of input vectors, $X_{p i}$ is the input vector variable of $\mathrm{p}, W_{i j}$ and $V_{j}$ are the weight of connections, $B_{j}$ and $B$ are the Bias amount of units and $O_{p}$ is the amount of network's output. The $f(x)=1 /(1+\exp (-x))$ is considered as the activation function of the neural network $[7,8]$.

\subsection{MLP}

Here an artificial neural network is adapted. The architecture of the network is given as follows:

Neuron configuration: 2-2-1

Number of hidden layer: one layer

Neurons in hidden layer: 2

Transfer function of hidden layer: sigmoid

Transfer function of output layer: linear

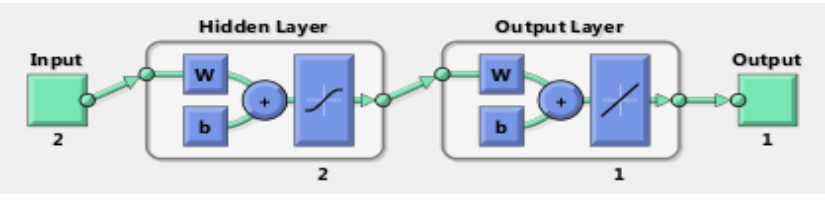

Figure 2. Network architecture in Matlab $\mathbb{R}$

Weight initialization: random

Performance criterion: Mean square of error (MSE)

Number of epoch: 76

MLP training algorithm: Back propagation (BP) which is an abbreviation for "backward propagation of error [8].

\subsection{Training Set}

Totally 126 observations along 16 years have been gathered through field (reservoir engineering) surveys. 75 percent of total data used for training the neural network. The results of training are given below:

As it is indicated, 99 percent accuracy in training procedure is given. A great match between output and target data have seen. Also, the error function is a normal identical distribution. The mean of error is almost zero with a small amount of variance. Moreover, the error function has a random distribution which shows a great manner.
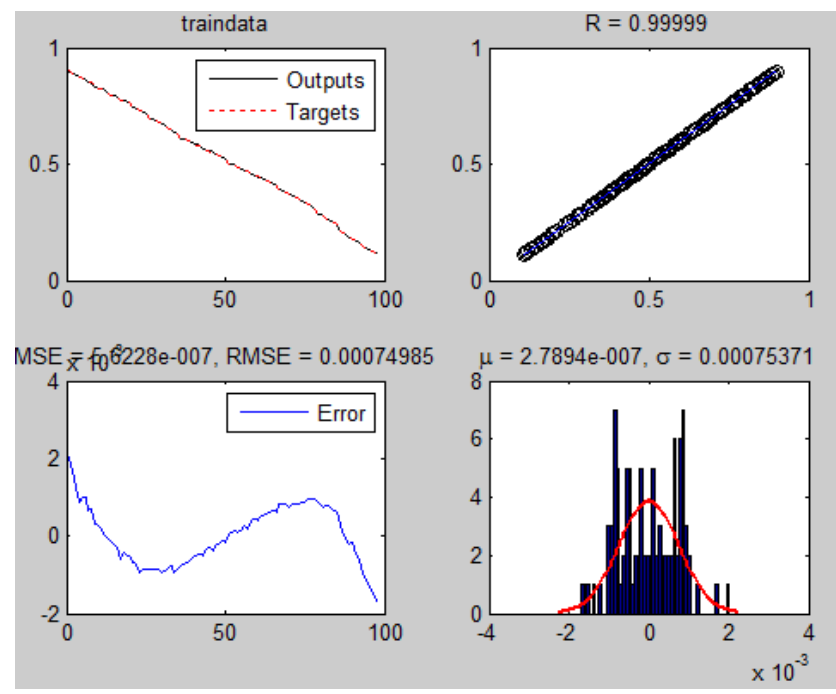

Figure3. Training data obtained in Matlab ${ }^{\circledR}$

\subsection{Test Set}

For the remaining data, trained neural network receive a set of data which has never seen previously. In figure4, the test set is provided. 

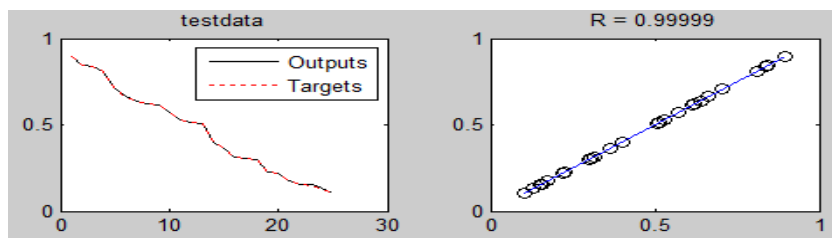

MSE $\overline{\times}=102503 \mathrm{e}-007, \mathrm{RMSE}=0.00085149$
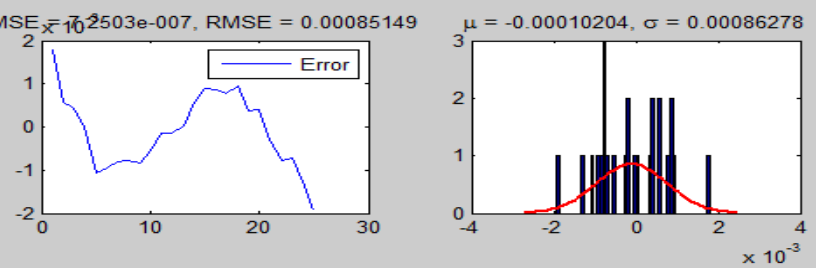

Figure4. Analysis of test data

\subsection{All Data}

Here all the data series are presented in figure 5.
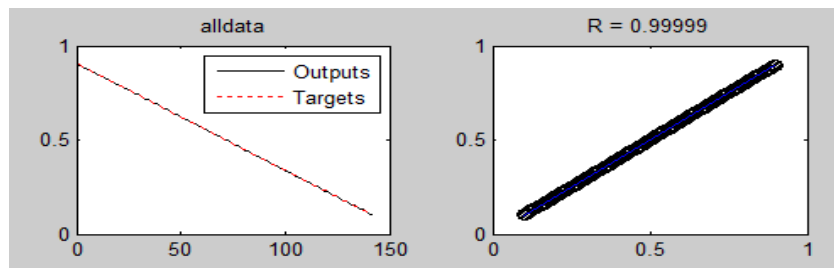

MSE $\bar{x}$ fo $\$ 379 \mathrm{e}-007, \mathrm{RMSE}=0.00078345$
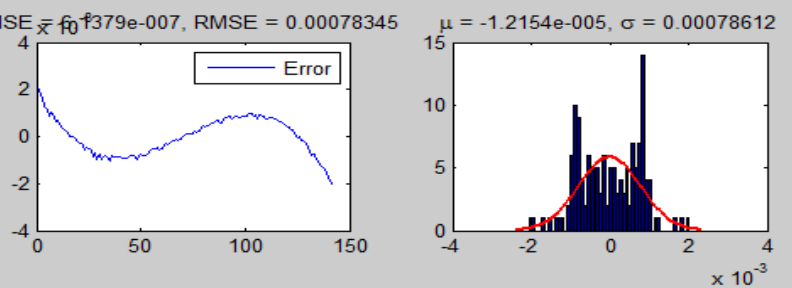

Figure5. The obtained results for all data

The above mentioned figure indicates the problem is well fitted. In the above figures, $\mathrm{R}=0.99$ indicates 99 percentage of accuracy are given ( both $\mathrm{X}$ and $\mathrm{Y}$ axis are shown $\mathrm{R}$ ). The output and targets have been closely matched to each other indicating a great matching between neural network and actual data has been found (number of observations versus accuracy). The error distribution follows an identical normal distribution function which indicates the error behavior is normal. Finally, error trend has a stochastic fluctuation indicating this is a random process. (Error amount in terms of observations)

Here, applying individual neural network is in-sufficient in context. That is why; UGS faces with many un-known parameters to be discovered by time series analysis according to the gathered data is different time intervals.

\section{Time Series Analysis}

As time passes, pressure trend will gradually drop. This decline depends on time and production rate. Here a time series model is given to model pressure. When pressure reach to 140 bar, well abandonment will happened.

$$
\text { Pressure }(\mathrm{t})=362-0.581 * \text { Time- } 2.28 * \operatorname{Rate}(\mathrm{t})
$$

Analysis of variance for equation 1 is given below:

Table 1. Analysis of variance

\begin{tabular}{|c|c|c|c|c|}
\hline Predictor & Coef & SE Coef & T & P \\
\hline Constant & 362.131 & 1.308 & 276.85 & 0.000 \\
\hline Time & -0.581306 & 0.0086 & -67.07 & 0.000 \\
\hline Rate & -2.2794 & 0.5534 & -4.12 & 0.000 \\
\hline
\end{tabular}

The p-value equals to zero indicates the model is well fitted.

The above mentioned equation shows at period of 375 reservoir abandonment is expected to happen.The results show the model predicts totally 370 Mcfduring more than 12 years. Average predicted amount of production rate is as of 2.6 MCF. The production forecasting rate has a decline trend which shows Average 1.8 MCF for the next year( the average actual production rate is equal to $3 \mathrm{MCF}$ ). This amount will significantly decrease after passing time and when it is near to abandonment (at period 375). For the last year average production rate is equal to $0.5 \mathrm{MCF}$ and at the last period the reservoir production is forecasting as $0.2 \mathrm{MCF}$. Therefore, depletion rate of UGS can be determined and therefore working gas amount can be then estimated for further calculations in UGS. It means that as time passes, the production rate will decline and instead it is possible to inject gas into underground reservoirs. Further calculations maybe made on economic analysis for each scenarios based on the obtained prediction of production rates.

The approach can be used in practice where collection of data is very time consuming and costly work to perform or traditional modeling procedures are not possible or maybe alternative approaches simultaneously developed to cover up whole the situations through an integrated approach.

\section{Comparative Analysis}

Here different scenarios for neural network models after running in Matlab ${ }^{\circledR}$ software are summarized below:

Table 2. Comparative analysis

\begin{tabular}{|c|c|c|}
\hline $\begin{array}{c}\text { Multi layer perception } \\
\text { neural networks } \\
\text { (MLP) }\end{array}$ & Percent of accuracy & Error function \\
\hline $\begin{array}{c}\text { Generalized regression } \\
\text { neural networks } \\
(\text { GRNN) }\end{array}$ & $96 \%$ & Normal \\
\hline
\end{tabular}

The generalized regression neural networks (GRNN) falls into probabilistic category of neural modeling that applies normal distribution (probability distribution density) as transfer function. This type of network is extended models of Radial basis neural networks and had been extensively applied through the literature [7]. After comparison of MLP 
and GRR it is found both networks have a normal distribution for error function, however, MLP shown a better performance especially in test area, when the data for the first time have been examined. Therefore, MLP is selected as the best known model with 99 percent of accuracy. Moreover, as long as the MLP shown a great performance in training and test area there is no need to put additional efforts to compare the results with other commonly used neural networks. The results seem to be satisfactory at this stage and further collaboration will be made through extension of UGS in another aspects.

\section{Conclusion Remark and Further Recommendation}

In this paper, several types of neural networks have been evaluated for forecasting of underground gas storage. The best fitness has been occurred with MLP neural network. Also, by incorporating a time series analysis and embedding with the fitted neural network model, prediction has been appropriately performed. Statistical analysis and p-value approved our hypothesis indicating the model is well fitted. This approach would be significantly beneficial for forecasting of working gas in UGS. Further research can be made on applying simulation based optimization through neural network modeling to tune the network architecture. Additionally, economic evaluation of the developed scenarios can be further elaborated.

\section{REFERENCES}

[1] US energy information administration, Independent and statistics report, August 2004.

[2] Underground gas storage, Wikipedia, the free encyclopedia.

[3] J.W. Duane, Gas storage field development optimization, Journal of Petroleum Technology, 19(3), 1967, 323-330.

[4] R.A. Cooksey, J.H. Henderson, \& J.R. Dempsey, Totalcomputer simulation of gas producing complex, Journal ofPetroleum Technology, 21(8), 1969, 942-948.222

[5] L. Ayala H, T. Ertekin, \& M. Adewumi, Study of gas-condensate reservoir exploitation using neuro-simulation, SPEReservoir Engineering and Evaluation, 10(2), 2007, 140-149

[6] A.W. Mann., A.F.Ayala, intelligent design and modeling of natural gas storage reservoirs, international journal of modeling and simulation,2009.

[7] E.Abounoori ,M.Bagherpour, “ Industrial cost estimation using neural network, regression analysis or hybrid regression- neural networks"? Iranian Economic Review, Vol 70, PP. 70-53. 5770 .

[8] MR. Feylizadeh, A. Hendalianpour, M. Bagherpour, A fuzzy neural network to estimate at completion costs of construction projects, International Journal of Industrial Engineering Computations, 5775, 2012. 